Supplement of Atmos. Chem. Phys. Discuss., 14, 17067-17099, 2014

http://www.atmos-chem-phys-discuss.net/14/17067/2014/

doi:10.5194/acpd-14-17067-2014-supplement

(C) Author(s) 2014. CC Attribution 3.0 License.

(c) (i)

Supplement of

\title{
Amino acids in Antarctica: evolution and fate of marine aerosols
}

\author{
E. Barbaro et al.
}

Correspondence to: E. Barbaro (barbaro@unive.it) 


\section{Reagents and standard solutions}

Ultra grade methanol (MeOH) was purchased from Romil LTD (Cambridge, UK), while Ultrapure water (18.2 M $\Omega, 0.01$ TOC) was produced using a Purelab Ultra System (Elga, High Wycombe,UK). Formic acid ( $\geq 98 \%)$ eluent additive for HPLC system was obtained from Fluka (Sigma Aldrich®, Buchs, Switzerland) and hydrochloric acid (HCl) 37\% ACS was supplied by Carlo Erba.

Each amino acid standard solution (D and L-alanine (D-/L-Ala), D and L-arginine (D-/L-Arg), D and L - asparagine (D-/L-Asn), D and L-aspartic acid (D-/L-Asp), D and L -glutamic acid (D-/LGlu), glycine (Gly), D and L hydroxy proline (D-/L-Hyp), D and L -histidine (D-/L-Hys), D and L -isoleucine (D-/L-Ile), D and L - leucine (D-/L-Leu),D and L -methionine (D-/L-Met), D/L methionine sulfone $\left(\mathrm{MetSO}_{2}\right.$ ), L ornithine (L-Orn), D and L -phenylalanine (D-/L-Phe), L-proline (L-Pro), D and L -serine (D-/L-Ser), D and L -threonine (D-/L-Thr), D and L -tyrosine (D-/L-Tyr), D and L tryptophan (D-/L-Trp), and D and L -valine(D-/L-Val)) was prepared by solid standard (purity $\geq 98 \%$ ), diluted in $\mathrm{HCl} 0.1 \mathrm{M}$. The solid standards were purchased from Sigma Aldrich®. Isotopically-labeled $13 \mathrm{C}$ amino acids $\left(\mathrm{L}-\left[{ }^{13} \mathrm{C}_{3}\right]\right.$ alanine (Ala*), L- $\left[{ }^{13} \mathrm{C}_{4}\right]$ aspartic acid (Asp*), L$\left[{ }^{13} \mathrm{C}_{5}\right]$ glutamic acid $\left(\mathrm{Glu}^{*}\right)$, and L- $\left[{ }^{13} \mathrm{C}_{6}\right]$ arginine (Arg*); purity of $98 \%$ ) were purchased from Sigma Aldrich while L- $\left[{ }^{13} \mathrm{C}_{1}\right]$ leucine $\left(\mathrm{Leu}^{*}\right)$, L- $\left[{ }^{13} \mathrm{C}_{1}\right]$ phenylalanine (Phe*), L- $\left[{ }^{13} \mathrm{C}_{1}\right]$ proline (Pro*), and $\mathrm{L}-\left[{ }^{13} \mathrm{C}_{1}\right]$ valine $\left(\mathrm{Val}^{*}\right)$ (purity $\geq 98 \%$ ) were obtained from Cambridge Isotope Laboratories Inc. (Andover, MA).

\section{Quality control}

The main aim of our work was to obtain a unique pre-analytical procedure to determine multiple analytes in the same samples.

The analytical procedure was evaluated through the estimation of trueness, repeatability and efficiency (yield\%) of the sample treatment process, in order to apply it to the enantiomeric determination of amino acids. This evaluation was performed by spiking five cleaned quartz filters (for each type of filter) with $100 \mu \mathrm{L}$ of native $\mathrm{L}$ and $\mathrm{D}$ amino acids solution (concentrations ranging between 2 and $4 \mu \mathrm{g} \mathrm{mL}{ }^{-1}$ ) and $100 \mu \mathrm{L}$ of isotopically-labeled ${ }^{13} \mathrm{C}$ amino acids solution (concentration ranging between 2 and $3 \mu \mathrm{g} \mathrm{mL}{ }^{-1}$ ). The filters were subsequently extracted as described in the section "Sample processing".

Tables S2, S3 and S4 report a summary of yields, trueness and relative standard deviation $(\mathrm{n}=5)$ for each type of filter used in this study. 
Average yields of $61 \%, 56 \%$ and $56 \%$ were obtained with circular, slotted and background filtersrespectively. In some cases, these values are lower than those reported in the literature (Barbaro et al., 2011;Mandalakis et al., 2010).

Trueness is the most important parameter during method validation; it refers to the degree of closeness of the determined value to the known "true" value. It is expressed as an error \%, calculated as $(\mathrm{Q}-\mathrm{T}) / \mathrm{T} \times 100$, where $\mathrm{Q}$ is the determined value and $\mathrm{T}$ is the "true value".

For circular filters, all D- and L-amino acids considered in this work were validated with an error percentage ranging between $-13 \%$ (D-Leu/D-Ile) and $+8 \%$ (L-Tyr).

In background filters, only D- and L-Hys produced unacceptable errors $\%$, and for this reason these compounds were excluded from the quantification. The other amino acids considered in this study were quantified with an accuracy ranging between $-9 \%$ (D-Met) and $+9 \%$ (D-Ala, L-Thr).

Some amino acids (D-Ala, L-Asn, D-Asn, D-Glu, D-Phe, L-Ser, D-Ser, and D-Val) were excluded from the quantification using the slotted quartz fiber filters because very high errors percentages were calculated. This behavior is probably due to different material of sampling support: slotted quartz fiber filters were used as impact supports while the other supports were filters. The preanalytical procedures were validated for other amino acids studied in this work with error \% values between $-13 \%$ (D-Tyr) and +13\% (D-Leu/D-Ile).

Repeatability estimated as relative standard deviation with 5 five tests for each type of filters was always below $10 \%$.

The method detection limit (MDL) for the analytical procedure is defined as three times the standard deviation of the average values of the field blank $(n=3)$. Tables S2, S3 and S4 report the relative MDLs for each quantified amino acid in three different sampling supports.

The comparison between previously published data and the MDLs obtained for each type of filters in this work showed lower values than the best MDLs reported in literature by Barbaro et al. (Barbaro et al., 2011) and Matsumoto and Uematsu (Matsumoto and Uematsu, 2005). 
Table S1. Position of R/V Italica on the Southern Ocean during the austral summer field 2012 and sampling volumes for each sample.

\begin{tabular}{|c|c|c|c|c|c|c|c|c|c|}
\hline & UTC date & UTC hour & \multicolumn{3}{|c|}{ latitude } & \multicolumn{3}{|c|}{ longitude } & $\begin{array}{c}\text { sampling } \\
\text { volume }\left(\mathrm{m}^{3}\right)\end{array}$ \\
\hline \multirow{6}{*}{ Sample 1} & 13 Jan & 3.00 & $44^{\circ}$ & $41^{\prime}$ & $S$ & $173^{\circ}$ & 32 & $\mathrm{E}$ & \multirow{6}{*}{511} \\
\hline & 14 Jan & 3.00 & $49^{\circ}$ & $23^{\prime}$ & $S$ & $173^{\circ}$ & $14^{\prime}$ & $\mathrm{E}$ & \\
\hline & 15 Jan & 3.00 & $53^{\circ}$ & $48^{\prime}$ & $\mathrm{S}$ & $173^{\circ}$ & $19^{\prime}$ & $\mathrm{E}$ & \\
\hline & $16 \mathrm{Jan}$ & 3.00 & $56^{\circ}$ & $35^{\prime}$ & $\mathrm{S}$ & $172^{\circ}$ & $46^{\prime}$ & $\mathrm{E}$ & \\
\hline & 17 Jan & 3.00 & $60^{\circ}$ & $1^{\prime}$ & $\mathrm{S}$ & $172^{\circ}$ & $61^{\prime}$ & $\mathrm{E}$ & \\
\hline & $18 \mathrm{Jan}$ & 13.00 & $63^{\circ}$ & $44^{\prime}$ & $S$ & $173^{\circ}$ & $54^{\prime}$ & $E$ & \\
\hline \multirow{6}{*}{ Sample 2} & $18 \mathrm{Jan}$ & 22.30 & $65^{\circ}$ & $33^{\prime}$ & S & $175^{\circ}$ & $13^{\prime}$ & $\mathrm{E}$ & \multirow{6}{*}{1386} \\
\hline & 19 Jan & 9.00 & $66^{\circ}$ & $22^{\prime}$ & $S$ & $179^{\circ}$ & $60^{\prime}$ & $\mathrm{E}$ & \\
\hline & $20 \mathrm{Jan}$ & 22.00 & $68^{\circ}$ & 53 & $\mathrm{~S}$ & $166^{\circ}$ & $50^{\prime}$ & W & \\
\hline & $21 \mathrm{Jan}$ & 22.00 & $74^{\circ}$ & $21{ }^{\prime}$ & $\mathrm{S}$ & $166^{\circ}$ & $42^{\prime}$ & W & \\
\hline & $22 \mathrm{Jan}$ & 16.00 & $74^{\circ}$ & $51^{\prime}$ & $\mathrm{S}$ & $179^{\circ}$ & 60 & W & \\
\hline & $23 \mathrm{Jan}$ & 9.00 & $75^{\circ}$ & $10^{\prime}$ & $S$ & $167^{\circ}$ & $18^{\prime}$ & $\mathrm{E}$ & \\
\hline \multirow{5}{*}{ Sample 3} & $25 \mathrm{Jan}$ & 9.00 & $75^{\circ}$ & $47^{\prime}$ & S & $166^{\circ}$ & '’ & $\mathrm{E}$ & \multirow{5}{*}{927} \\
\hline & $26 \mathrm{Jan}$ & 9.00 & $72^{\circ}$ & $24^{\prime}$ & $S$ & $172^{\circ}$ & $58^{\prime}$ & $\mathrm{E}$ & \\
\hline & 27 Jan & 9.00 & $73^{\circ}$ & 60 ' & $S$ & $175^{\circ}$ & 6 & $\mathrm{E}$ & \\
\hline & $28 \mathrm{Jan}$ & 9.00 & $73^{\circ}$ & $52^{\prime}$ & $S$ & $174^{\circ}$ & $38^{\prime}$ & $\mathrm{E}$ & \\
\hline & 29 Jan & 13.00 & $74^{\circ}$ & $41^{\prime}$ & $S$ & $164^{\circ}$ & 8 & $\mathrm{E}$ & \\
\hline \multirow{7}{*}{ Sample 4} & 31 Jan & 11.00 & $74^{\circ}$ & $44^{\prime}$ & S & $164^{\circ}$ & $54^{\prime}$ & $\mathrm{E}$ & \multirow{7}{*}{1602} \\
\hline & $1 \mathrm{Feb}$ & 11.00 & $75^{\circ}$ & $23^{\prime}$ & $S$ & $166^{\circ}$ & $20{ }^{\prime}$ & $\mathrm{E}$ & \\
\hline & $2 \mathrm{Feb}$ & 11.00 & $74^{\circ}$ & $55^{\prime}$ & $S$ & $168^{\circ}$ & $59^{\prime}$ & $\mathrm{E}$ & \\
\hline & $3 \mathrm{Feb}$ & 5.00 & $77^{\circ}$ & 13 & $\mathrm{~S}$ & $179^{\circ}$ & 60 & $\mathrm{E}$ & \\
\hline & $4 \mathrm{Feb}$ & 20.00 & $77^{\circ}$ & $47^{\prime}$ & $S$ & $179^{\circ}$ & $60^{\prime}$ & $\mathrm{W}$ & \\
\hline & $5 \mathrm{Feb}$ & 11.00 & $77^{\circ}$ & $24^{\prime}$ & $S$ & $172^{\circ}$ & $2^{\prime}$ & $\mathrm{E}$ & \\
\hline & 6 Feb & 18.00 & $77^{\circ}$ & $41^{\prime}$ & $S$ & $166^{\circ}$ & 3' & $\mathrm{E}$ & \\
\hline \multirow{3}{*}{ Sample 5} & $7 \mathrm{Feb}$ & 13.00 & $77^{\circ}$ & 27 & S & $165^{\circ}$ & $38^{\prime}$ & $\mathrm{E}$ & \multirow{3}{*}{481} \\
\hline & $8 \mathrm{Feb}$ & 13.00 & $74^{\circ}$ & 18 & $\mathrm{~S}$ & $170^{\circ}$ & $26^{\prime}$ & $\mathrm{E}$ & \\
\hline & 9 Feb & 19.00 & $74^{\circ}$ & $41^{\prime}$ & S & $164^{\circ}$ & $9^{\prime}$ & $\mathrm{E}$ & \\
\hline \multirow{7}{*}{ Sample 6} & $12 \mathrm{Feb}$ & 9.00 & $74^{\circ}$ & $42^{\prime}$ & S & $164^{\circ}$ & 7 & $\mathrm{E}$ & \multirow{7}{*}{2154} \\
\hline & $13 \mathrm{Feb}$ & 9.00 & $71^{\circ}$ & $42^{\prime}$ & $S$ & $178^{\circ}$ & $8^{\prime}$ & $\mathrm{E}$ & \\
\hline & $14 \mathrm{Feb}$ & 9.00 & $66^{\circ}$ & $45^{\prime}$ & $\mathrm{S}$ & $178^{\circ}$ & 25 & $\mathrm{E}$ & \\
\hline & $15 \mathrm{Feb}$ & 9.00 & $62^{\circ}$ & 22 & $\mathrm{~S}$ & $179^{\circ}$ & 56 & $\mathrm{E}$ & \\
\hline & $16 \mathrm{Feb}$ & 9.00 & $58^{\circ}$ & $33^{\prime}$ & $S$ & $179^{\circ}$ & $0^{\prime}$ & $\mathrm{E}$ & \\
\hline & $17 \mathrm{Feb}$ & 9.00 & $54^{\circ}$ & $10^{\prime}$ & $S$ & $177^{\circ}$ & $45^{\prime}$ & $\mathrm{E}$ & \\
\hline & $18 \mathrm{Feb}$ & 6.23 & $51^{\circ}$ & $47^{\prime}$ & $S$ & $176^{\circ}$ & $24^{\prime}$ & $E$ & \\
\hline \multirow{2}{*}{ Sample 7} & $18 \mathrm{Feb}$ & 5.00 & $51^{\circ}$ & $47^{\prime}$ & S & $176^{\circ}$ & $24 '$ & $\mathrm{E}$ & \multirow{2}{*}{520} \\
\hline & $19 \mathrm{Feb}$ & 15.00 & $43^{\circ}$ & $45^{\prime}$ & $S$ & $173^{\circ}$ & $14^{\prime}$ & $\mathrm{E}$ & \\
\hline
\end{tabular}


Table S2. Summary of yield, trueness and reproducibility expressed as relative standard deviation (RSD\%) for circular quartz fiber filters. For each amino acid, the internal standard (IS) used for the quantification and the relative method detection limit (MDL)is reported.

\begin{tabular}{|c|c|c|c|c|c|c|}
\hline & IS & $\begin{array}{c}\text { Yield } \\
\%\end{array}$ & $\begin{array}{c}\text { RSD } \\
\%\end{array}$ & $\begin{array}{c}\text { Error } \\
\%\end{array}$ & $\begin{array}{c}\text { RSD } \\
\%\end{array}$ & $\begin{array}{c}\text { MDL } \\
\text { (ng abs) }\end{array}$ \\
\hline L-Ala & Ala* & 51 & 10 & -10 & 2 & 44 \\
\hline D-Ala & Ala* & 62 & 7 & 4 & 3 & 22 \\
\hline L-Arg & Arg* & 62 & 8 & -2 & 9 & 2 \\
\hline D-Arg & Arg* & 78 & 2 & 0.7 & 1 & 3.2 \\
\hline L-Asn & Val* & 70 & 9 & 3 & 7 & 6 \\
\hline D-Asn & Val* & 67 & 2 & -4 & 9 & 6 \\
\hline L-Asp & Asp* & 50 & 8 & -7 & 6 & 30 \\
\hline D-Asp & Asp* & 41 & 8 & 2 & 13 & 50 \\
\hline L-Glu & Glu* & 58 & 7 & -6 & 2 & 6 \\
\hline D-Glu & Glu* & 57 & 9 & -1 & 6 & 8 \\
\hline L-Hyp & Leu* & 70 & 9 & 7 & 13 & 0.5 \\
\hline D-Hyp & Leu* & 74 & 7 & -5 & 10 & 1.4 \\
\hline L-Hys & Pro* & 66 & 9 & 6 & 1 & 16 \\
\hline D-Hys & Pro* & 49 & 7 & -8 & 8 & 10 \\
\hline L-Leu/L-Ile & Leu* & 60 & 5 & -7 & 6 & 32 \\
\hline D-Leu/D-Ile & Leu* & 59 & 5 & -13 & 6 & 5.8 \\
\hline L-Met & Leu* & 61 & 11 & -2 & 8 & 3.2 \\
\hline D-Met & Leu* & 65 & 9 & -9 & 3 & 10 \\
\hline L-Orn & Arg* & 43 & 13 & 2 & 14 & 58 \\
\hline L-Phe & Phe* & 63 & 5 & -3 & 4 & 2.8 \\
\hline D-Phe & Phe* & 70 & 4 & 7 & 9 & 5.8 \\
\hline L-Pro & Pro* & 61 & 9 & -4 & 3 & 3.8 \\
\hline L-Ser & Phe* & 38 & 17 & -2 & 8 & 138 \\
\hline D-Ser & Phe* & 56 & 15 & 6 & 13 & 84 \\
\hline L-Thr & Arg* & 65 & 6 & -1 & 5 & 5 \\
\hline D-Thr & Arg* & 72 & 6 & 5 & 6 & 1 \\
\hline L-Trp & Arg* & 63 & 10 & 5 & 6 & 5 \\
\hline D-Trp & Arg* & 65 & 10 & -0.2 & 9 & 1.2 \\
\hline L-Tyr & Phe* & 66 & 13 & 8 & 10 & 16 \\
\hline D-Tyr & Phe* & 67 & 9 & 7 & 10 & 8 \\
\hline L-Val & Val* & 61 & 6 & -6 & 2 & 12 \\
\hline D-Val & Val* & 64 & 10 & -5 & 5 & 3 \\
\hline Gly & Ala* & 71 & 11 & 3 & 4 & 50 \\
\hline MetSO2 & Leu* & 58 & 8 & -5 & 8 & 14 \\
\hline
\end{tabular}


Table S3. Summary of yield, trueness and reproducibility expressed as relative standard deviation (RSD\%) for backup quartz fiber filters $(<0.49 \mu \mathrm{m})$. For each amino acid, the internal standard (IS) used for the quantification and the relative method detection limit (MDL) is reported.

\begin{tabular}{|c|c|c|c|c|c|c|}
\hline & IS & $\begin{array}{c}\text { Yield } \\
\%\end{array}$ & $\begin{array}{c}\text { RSD } \\
\%\end{array}$ & $\begin{array}{c}\text { Error } \\
\%\end{array}$ & $\begin{array}{c}\text { RSD } \\
\%\end{array}$ & $\begin{array}{c}\text { MDL } \\
\text { (ng abs) }\end{array}$ \\
\hline L-Ala & Ala* & 51 & 6 & -8 & 3 & 35 \\
\hline D-Ala & Ala* & 61 & 9 & 9 & 7 & 36 \\
\hline L-Arg & Arg* & 53 & 7 & -7 & 2 & 24 \\
\hline D-Arg & Arg* & 61 & 8 & 8 & 3 & 3.0 \\
\hline L-Asn & $\mathrm{Val}^{*}$ & 55 & 6 & -2 & 6 & 28 \\
\hline D-Asn & $\mathrm{Val}^{*}$ & 54 & 9 & 6 & 9 & 28 \\
\hline L-Asp & Asp* & 54 & 6 & -2 & 4 & 35 \\
\hline D-Asp & Asp* & 54 & 9 & 1 & 8 & 28 \\
\hline L-Glu & Glu* & 54 & 5 & -3 & 4 & 18 \\
\hline D-Glu & Glu* & 50 & 9 & 5 & 7 & 8 \\
\hline L-Hyp & $\mathrm{Val}^{*}$ & 56 & 8 & 3 & 10 & 4 \\
\hline D-Нyp & $\mathrm{Val}^{*}$ & 70 & 8 & 7 & 7 & 5 \\
\hline L-Hys & Pro* & 57 & 32 & 77 & 15 & 114 \\
\hline D-Hys & Pro* & 46 & 16 & -21 & 10 & 64 \\
\hline L-Leu/L-Ile & Leu* & 57 & 3 & -8 & 9 & 23 \\
\hline D-Leu/D-Ile & Leu* & 61 & 9 & -4 & 2 & 12 \\
\hline L-Met & Leu* & 56 & 9 & -9 & 5 & 19 \\
\hline D-Met & Leu* & 51 & 5 & -9 & 2 & 6 \\
\hline L-Orn & Arg* & 42 & 5 & -3 & 8 & 61 \\
\hline L-Phe & Phe* & 60 & 7 & 1 & 6 & 11 \\
\hline D-Phe & Phe* & 65 & 9 & 4 & 8 & 2.0 \\
\hline L-Pro & Pro* & 55 & 5 & -4 & 3 & 42 \\
\hline L-Ser & Phe* & 55 & 7 & 8 & 5 & 167 \\
\hline D-Ser & Phe* & 42 & 9 & -6 & 5 & 61 \\
\hline L-Thr & Arg* & 63 & 10 & 9 & 1 & 18 \\
\hline D-Thr & Arg* & 58 & 5 & 7 & 3 & 9 \\
\hline L-Trp & Phe* & 63 & 9 & -5 & 7 & 4 \\
\hline D-Trp & Phe* & 61 & 9 & -1 & 9 & 10 \\
\hline L-Tyr & Phe* & 53 & 6 & 2 & 7 & 34 \\
\hline D-Tyr & Phe* & 62 & 11 & 7 & 8 & 8 \\
\hline L-Val & $\mathrm{Val}^{*}$ & 58 & 6 & -3 & 2 & 49 \\
\hline D-Val & $\mathrm{Val}^{*}$ & 50 & 8 & -7 & 8 & 12 \\
\hline Gly & Ala* & 56 & 6 & -1 & 3 & 37 \\
\hline MetSO2 & Leu* & 51 & 9 & -9 & 8 & 23 \\
\hline
\end{tabular}


Table S4. Summary of yield, trueness and reproducibility expressed as relative standard deviation (RSD\%) for slotted quartz fiber filters. For each amino acid, the internal standard (IS) used for the quantification and the relative method detection limit (MDL) is reported.

\begin{tabular}{|c|c|c|c|c|c|c|}
\hline & IS & $\begin{array}{c}\text { Yield } \\
\%\end{array}$ & $\begin{array}{c}\text { RSD } \\
\%\end{array}$ & $\begin{array}{c}\text { Error } \\
\%\end{array}$ & $\begin{array}{c}\text { RSD } \\
\%\end{array}$ & $\begin{array}{c}\text { MDL } \\
\text { (ng abs) }\end{array}$ \\
\hline L-Ala & Ala* & 72 & 6 & -6 & 5 & 24 \\
\hline D-Ala & Ala* & 346 & 6 & 450 & 7 & - \\
\hline L-Arg & Arg* & 51 & 7 & -9 & 4 & 4 \\
\hline D-Arg & Arg* & 53 & 3 & -1 & 6 & 1.1 \\
\hline L-Asn & $\mathrm{Val}^{*}$ & 32 & 5 & -64 & 5 & 0.2 \\
\hline D-Asn & $\mathrm{Val}^{*}$ & 190 & 7 & 191 & 9 & - \\
\hline L-Asp & Asp* & 71 & 6 & 1 & 3 & 51 \\
\hline D-Asp & Asp* & 37 & 26 & -3 & 33 & 107 \\
\hline L-Glu & Glu* & 81 & 2 & 7 & 4 & 1.1 \\
\hline D-Glu & Glu* & 494 & 10 & 799 & 9 & - \\
\hline L-Hyp & Leu* & 81 & 4 & 3 & 5 & 0.2 \\
\hline D-Нyр & Leu & 75 & 5 & -8 & 5 & 0.8 \\
\hline L-Hys & Leu* & 71 & 9 & 1 & 15 & 3 \\
\hline D-Hys & Asp* & 45 & 8 & -5 & 14 & 9 \\
\hline L-Leu/L-Ile & Leu* & 5 & 65 & 7 & -9 & 7 \\
\hline D-Leu/D-Ile & Leu* & 7 & 72 & 13 & -6 & 8 \\
\hline L-Met & Leu* & 61 & 10 & -8 & 11 & 4 \\
\hline D-Met & Leu* & 50 & 8 & -2 & 8 & 0.5 \\
\hline L-Orn & Arg* & 50 & 9 & 4 & 8 & 23 \\
\hline L-Phe & Phe* & 77 & 3 & -2 & 3 & 10 \\
\hline D-Phe & Phe* & 102 & 9 & 56 & 4 & - \\
\hline L-Pro & Pro* & 61 & 7 & -8 & 5 & 56 \\
\hline L-Ser & Phe* & 88 & 10 & 57 & 7 & - \\
\hline D-Ser & Phe* & -18 & -6 & -115 & -40 & - \\
\hline L-Thr & Arg* & 57 & 6 & -9 & 7 & 8 \\
\hline D-Thr & Arg* & 64 & 8 & 7 & 4 & 2 \\
\hline L-Trp & Phe* & 99 & 8 & 59 & 10 & - \\
\hline D-Trp & Phe* & 93 & 6 & 54 & 11 & - \\
\hline L-Tyr & Arg* & 61 & 4 & 6 & 4 & 16 \\
\hline D-Tyr & Arg* & 63 & 7 & -13 & 13 & 7 \\
\hline L-Val & $\mathrm{Val}^{*}$ & 78 & 3 & -3 & 3 & 12 \\
\hline D-Val & $\mathrm{Val}^{*}$ & 174 & 8 & 140 & 7 & - \\
\hline Gly & Ala* & 36 & 41 & -7 & 3 & 15 \\
\hline MetSO2 & Leu* & 66 & 5 & -9 & 5 & 4 \\
\hline
\end{tabular}




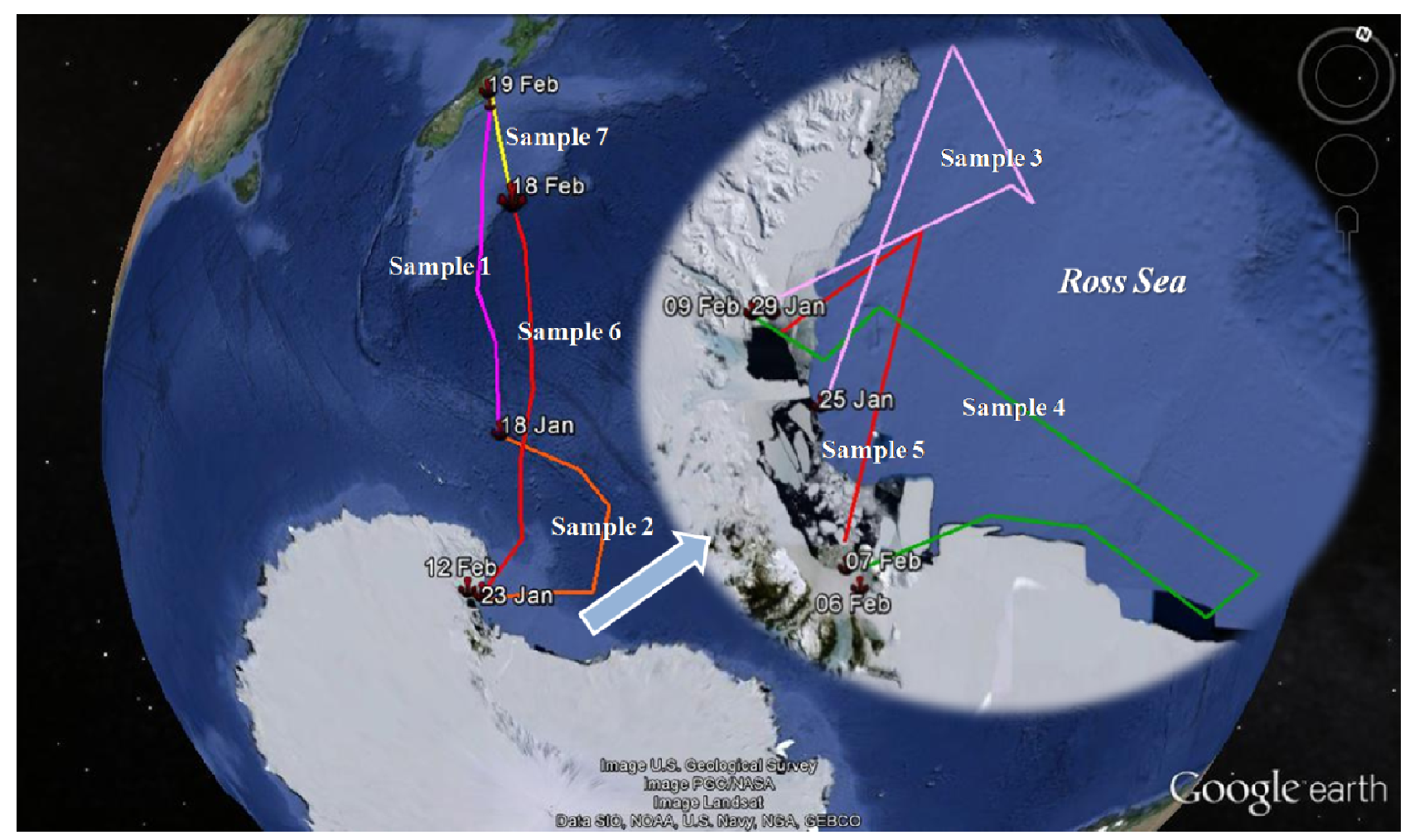

Figure S1. Cruise track of the R/V Italica on the Southern Ocean from New Zealand to Antarctica, cruise in the Ross Sea, and return to New Zealand from 13 January to 19 February 2012. In the circle, a zoom on the cruise track in the Ross Sea is reported. 
Cluster means - Standard 208 backward trajectories

CDC1 Meteorological Data

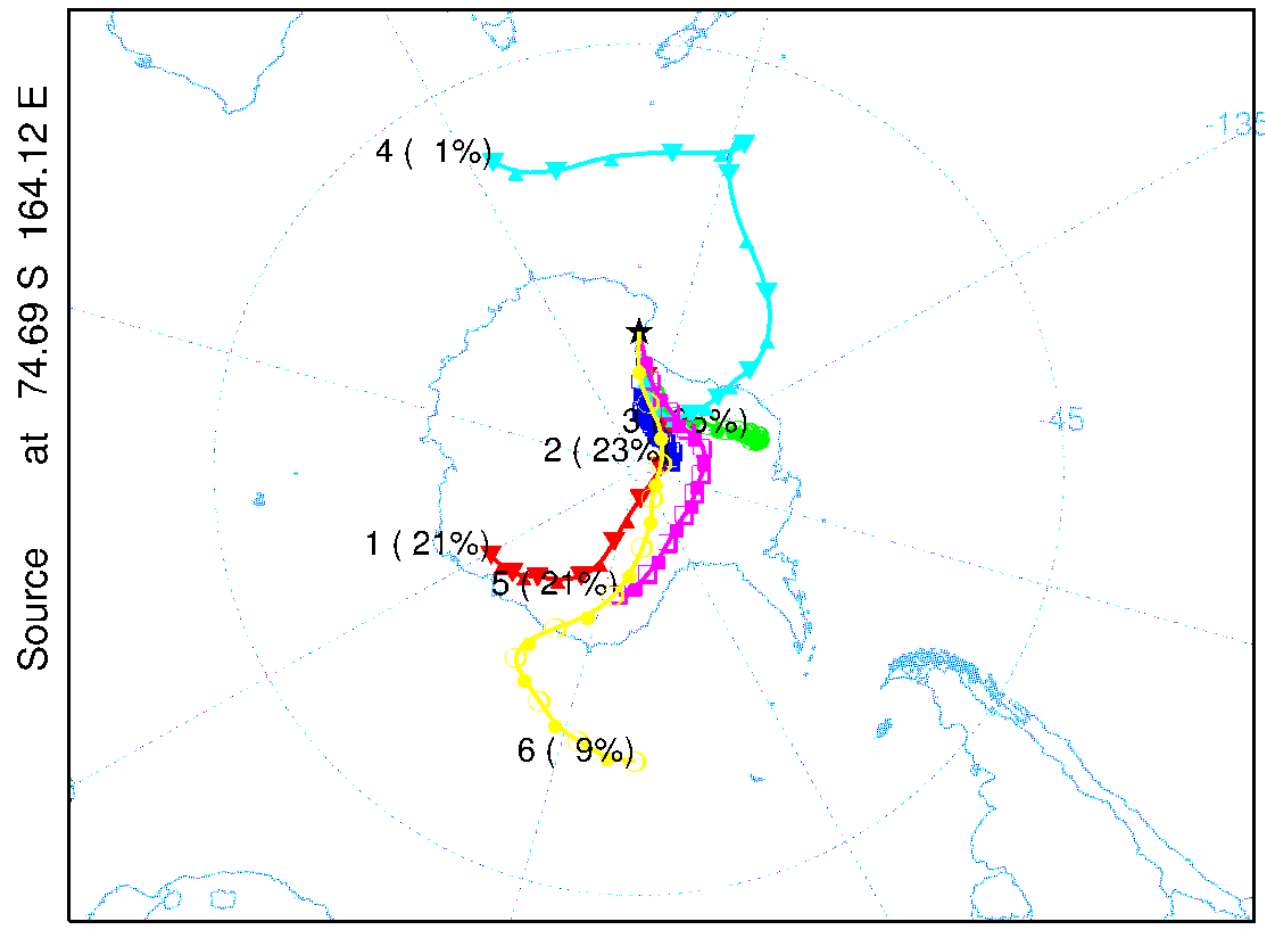

Figure S2. Cluster means backward trajectories analyses at $500 \mathrm{~m}$ agl for the period from $29^{\text {th }}$ November 2010 to $18^{\text {th }}$ January 2011 in the coastal base "Mario Zucchelli Station". 


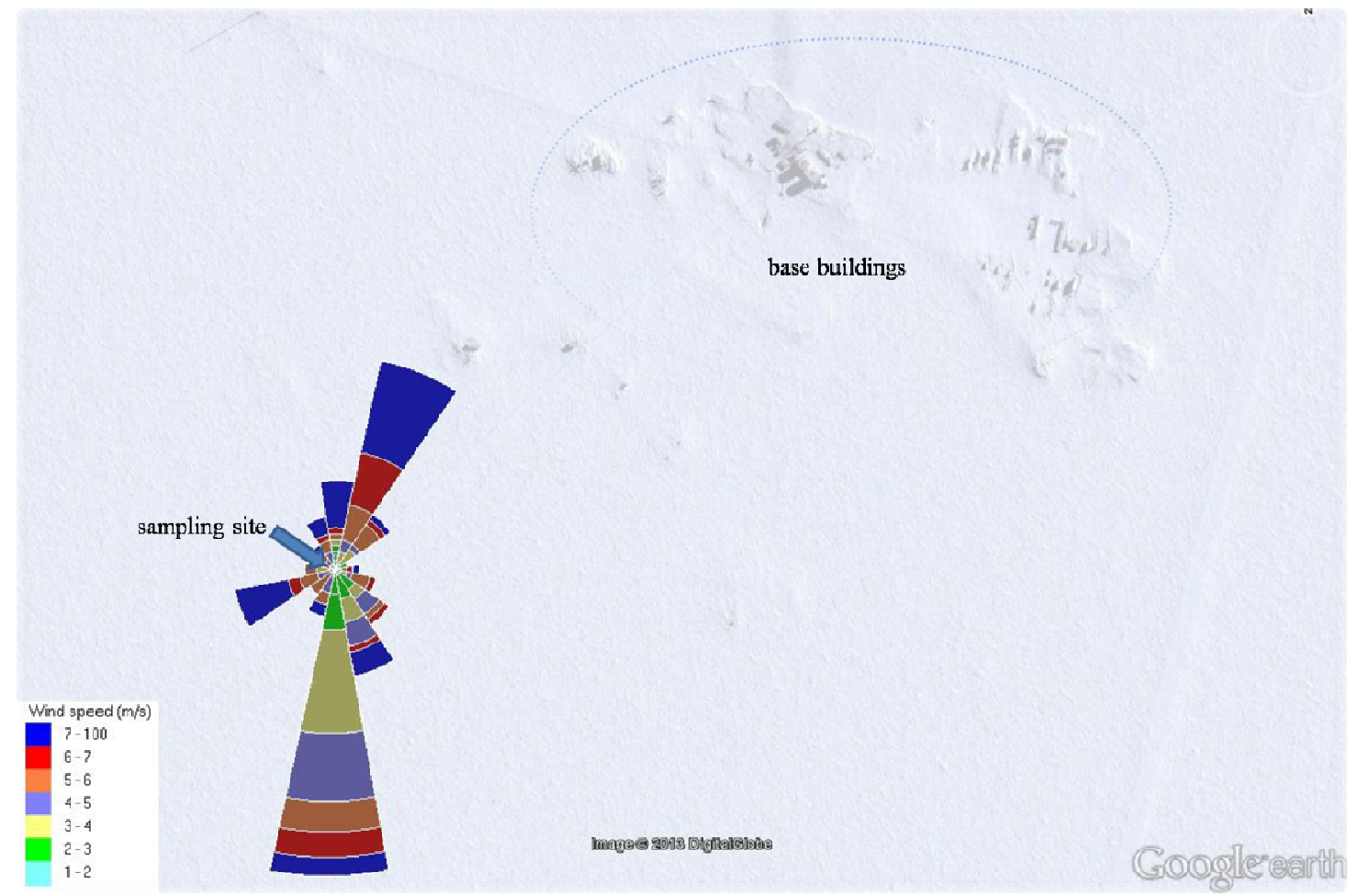

Figure S3. Wind rose of the sample collected from 27 December to 06 January at the Italian-French base "Concordia Station" (Dome C) during the austral summer Antarctica 2012-13. 


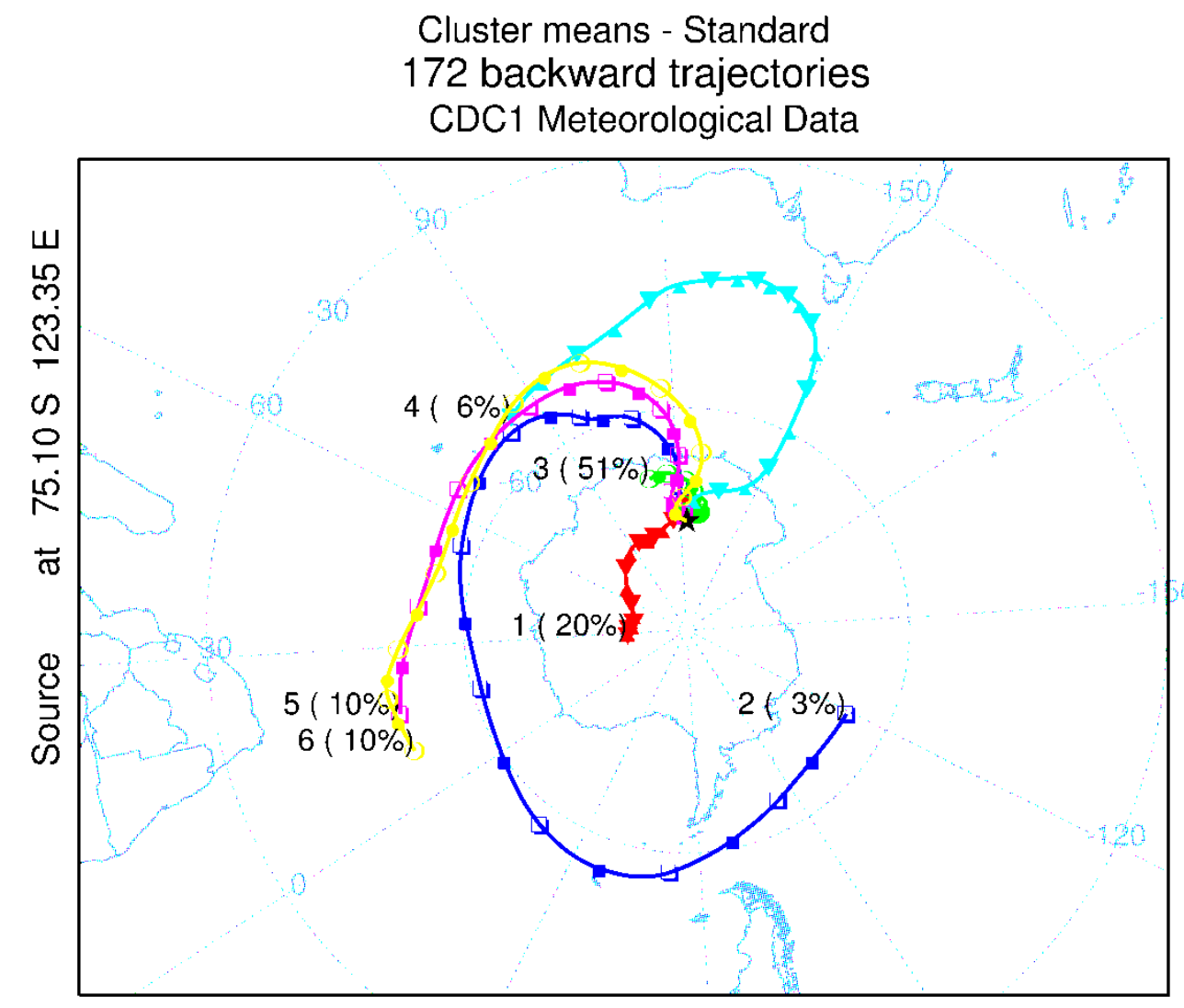

Figure S4. Cluster means backward trajectories analyses at $500 \mathrm{~m} \mathrm{agl}$ for the period from $19^{\text {th }}$ December 2011 to $30^{\text {th }}$ January 2012 in the Italian-French base "Concordia Station" (Dome C). 


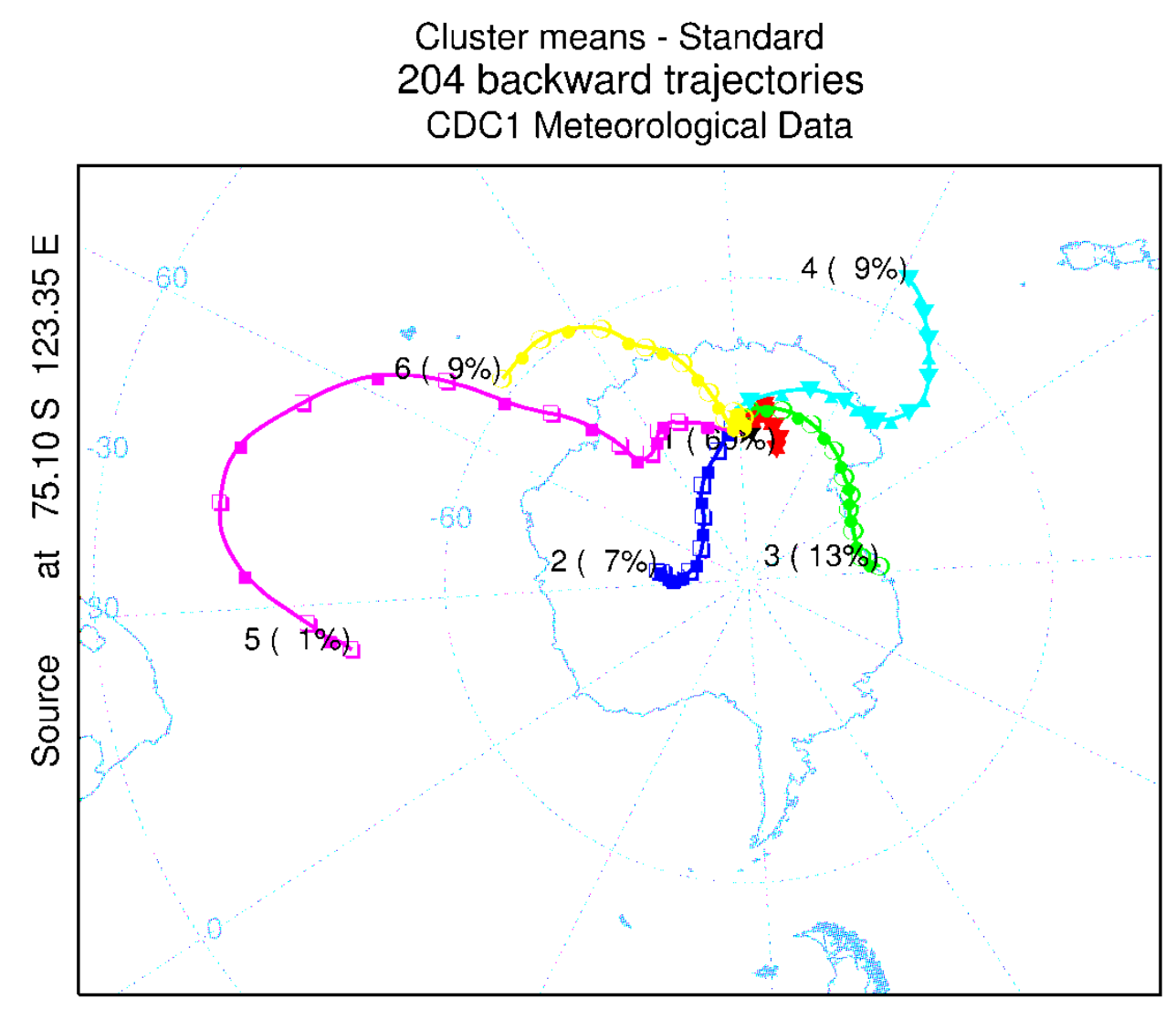

Figure S5. Cluster means backward trajectories analyses at $500 \mathrm{~m} \mathrm{agl}$ for the period from $7^{\text {th }}$ December 2012 to $26^{\text {th }}$ January 2013 in the Italian-French base "Concordia Station" (Dome C). 


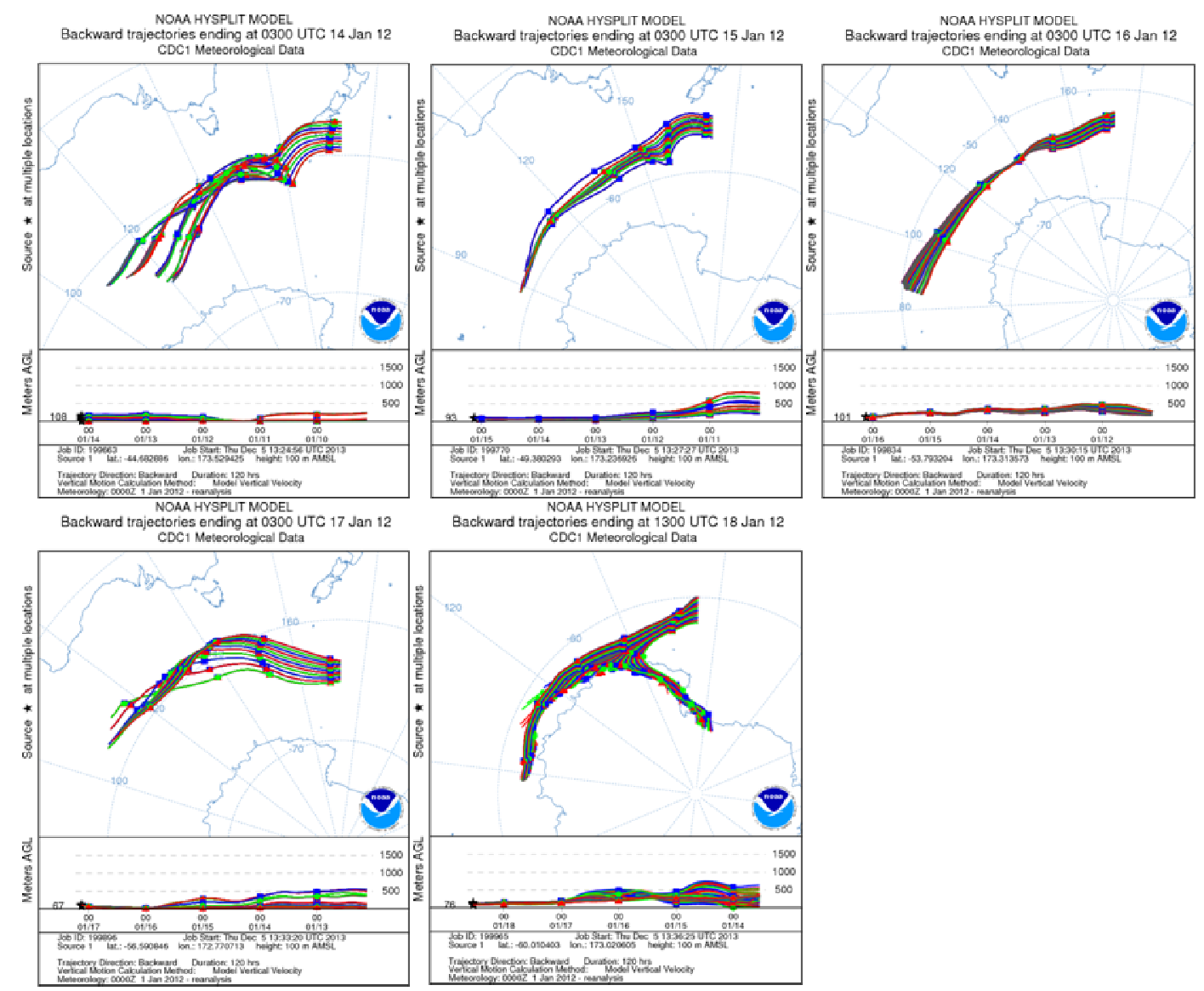

Figure S6A. 5-day back-trajectories of the first sample (from 13 to 18 January) collected during the oceanographic cruise on the R/V Italica during the austral summer Antarctica field 2011-12 on the Southern Ocean. The trajectories were computed for each $24-\mathrm{h}$ sampling using a vertical velocity model at $100 \mathrm{~m}$ above sea level. 


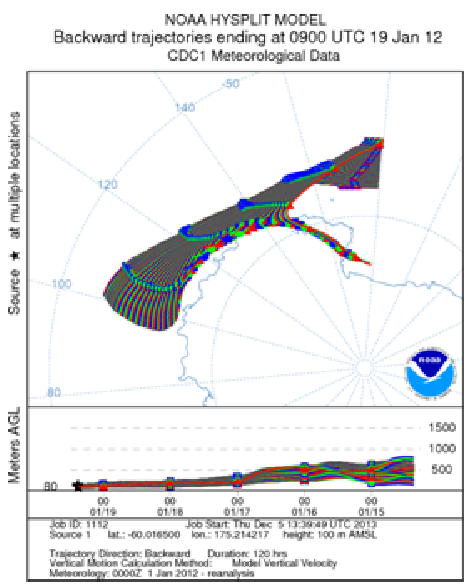

Nackward traiectom HYSPUT MODEL
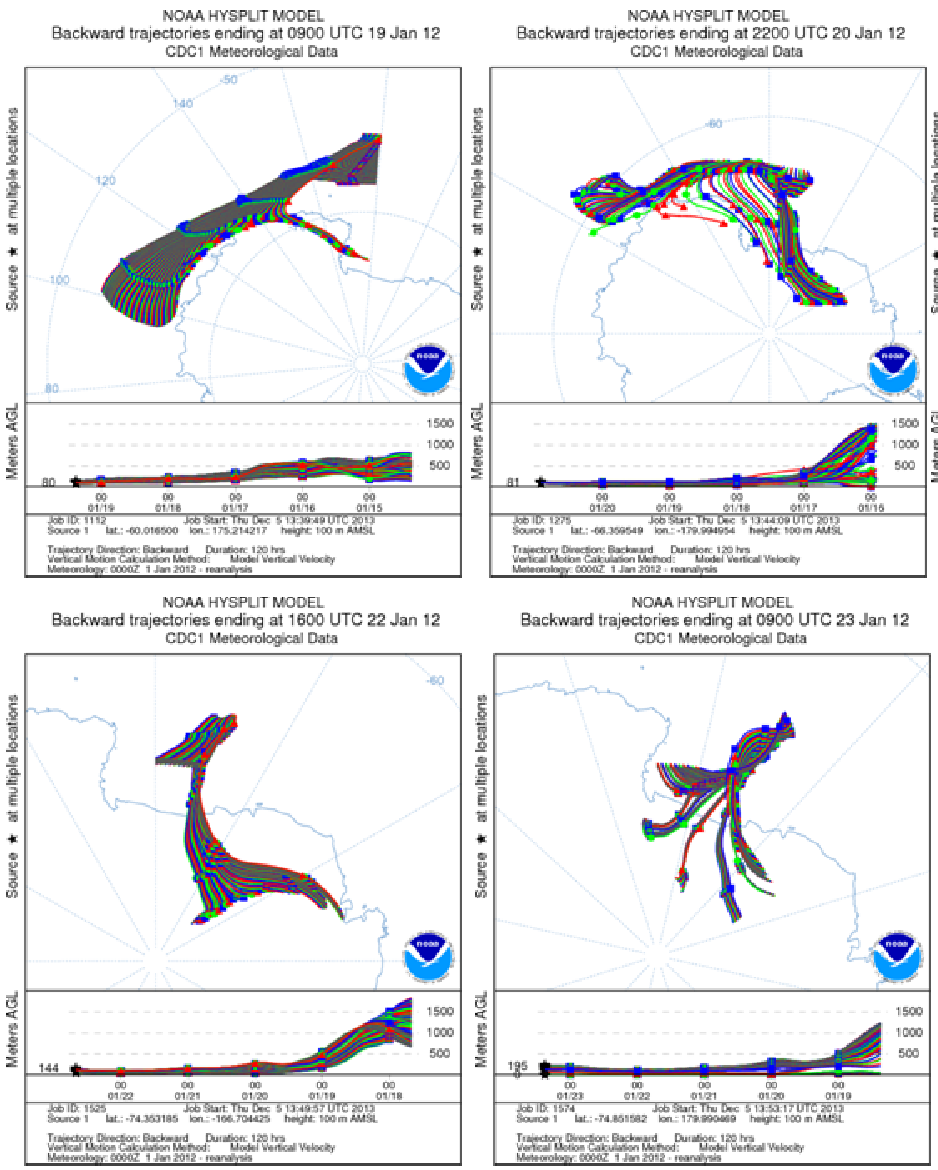

NOAA HYSPUT MODEL
Backward trajectories ending at 0900 UTC 23 Jan 12 CDC1 Meteorological Dat

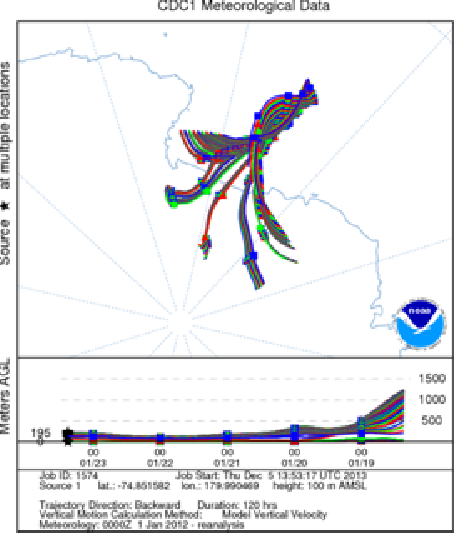

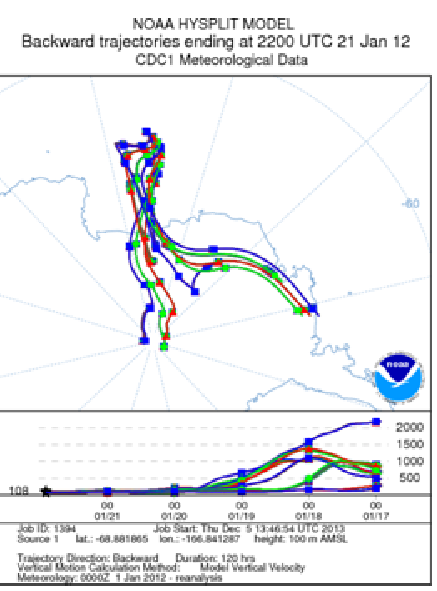

Figure S6B. 5-day back-trajectories of the second sample (from 18 to 23 January) collected during the oceanographic cruise on the R/V Italica during the austral summer Antarctica field 2011-12 on the Southern Ocean. The trajectories were computed for each 24-h sampling using a vertical velocity model at $100 \mathrm{~m}$ above sea level. 

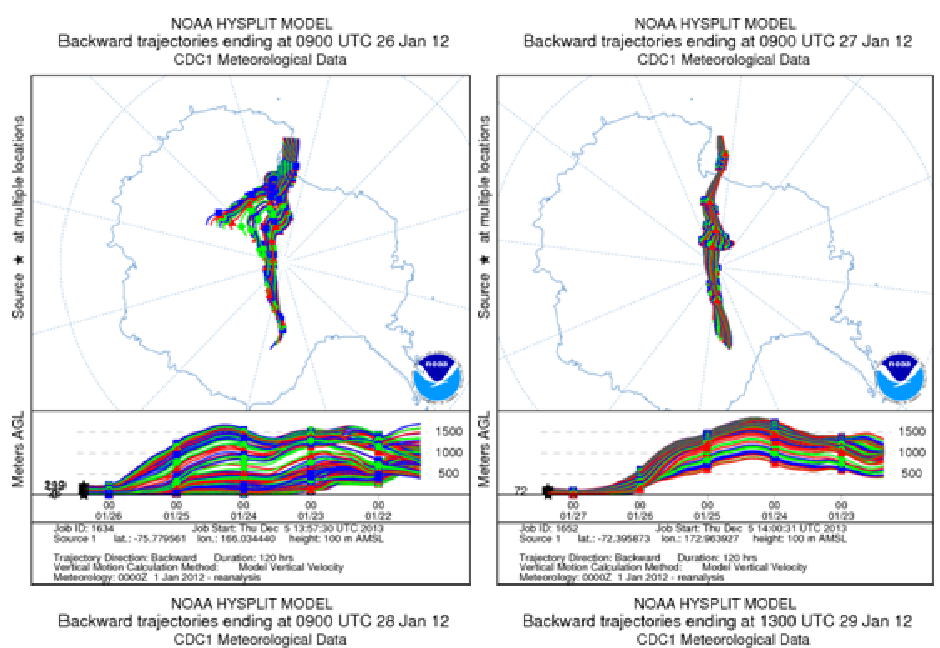

Backward trajectories ending at 1300 UTC 29 Jan 12
CDC1 Meteorological Data
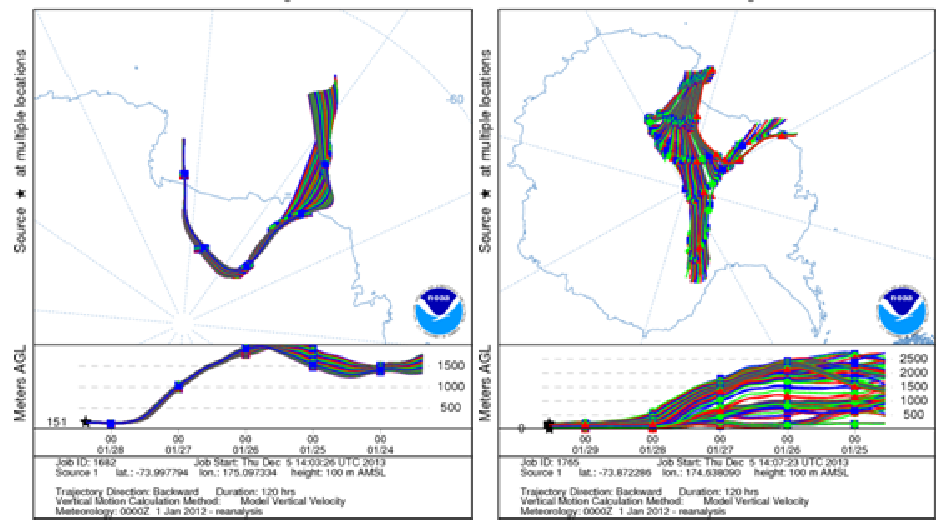

Figure S6C. 5-day back-trajectories of the third sample (from 25 to 29 January) collected during the oceanographic cruise on the R/V Italica during the austral summer Antarctica field 2011-12 on the Ross Sea. The trajectories were computed for each 24-h sampling using a vertical velocity model at $100 \mathrm{~m}$ above sea level. 

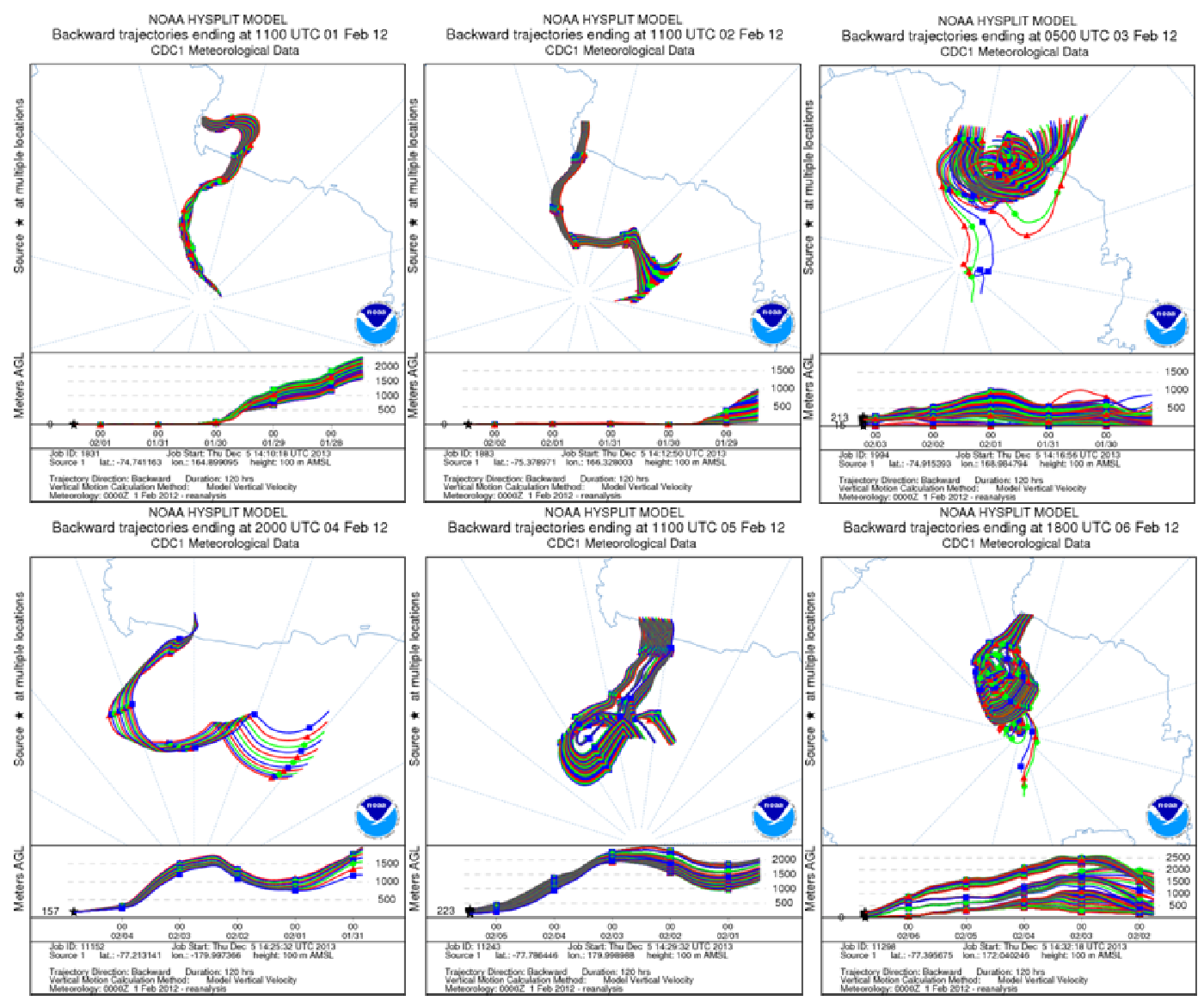

Figure S6D. 5-day back-trajectories of the fourth sample (from 31 January to 6 February) collected during the oceanographic cruise on the R/V Italica during the austral summer Antarctica field 201112 on the Ross Sea. The trajectories were computed for each 24-h sampling using a vertical velocity model at $100 \mathrm{~m}$ above sea level. 


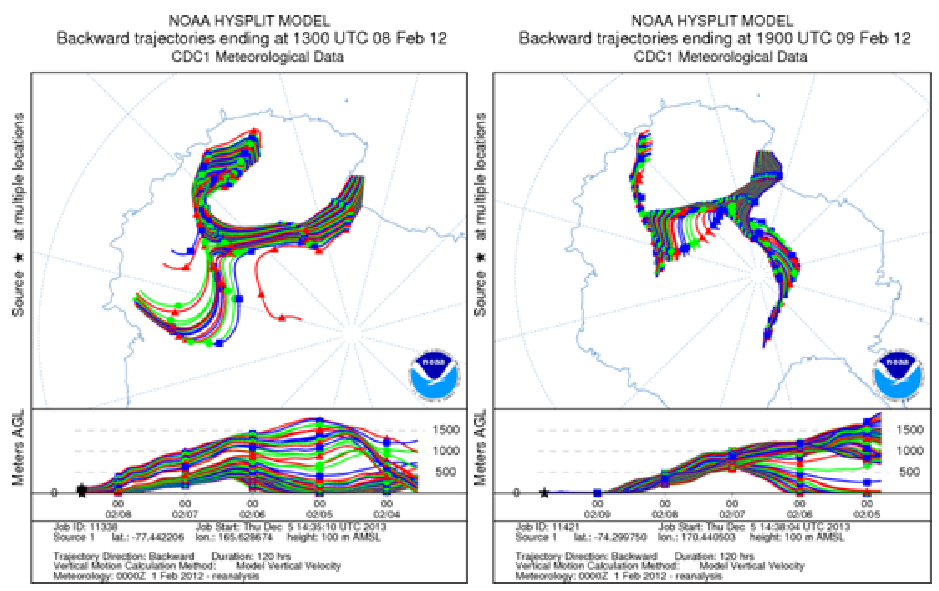

Figure S6E. 5-day back-trajectories of the fifth sample (from 7 to 9 February) collected during the oceanographic cruise on the R/V Italica during the austral summer Antarctica field 2011-12 on the Ross Sea. The trajectories were computed for each 24-h sampling using a vertical velocity model at $100 \mathrm{~m}$ above sea level.

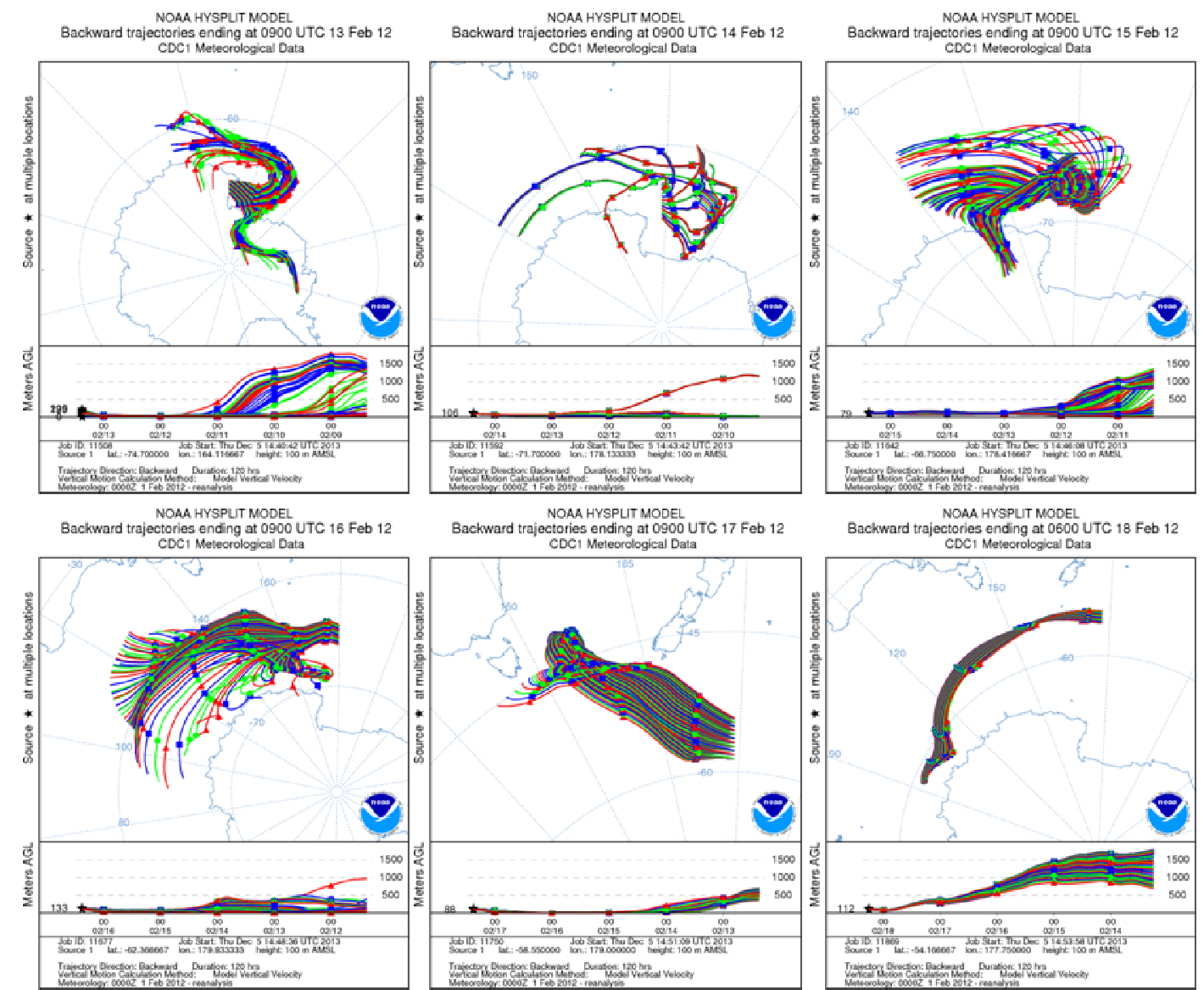

Figure S6F. 5-day back-trajectories of the sixth sample (from 13 to 18 February) collected during the oceanographic cruise on the R/V Italica during the austral summer Antarctica field 2011-12 on the Southern Ocean. The trajectories were computed for each 24-h sampling using a vertical velocity model at $100 \mathrm{~m}$ above sea level. 


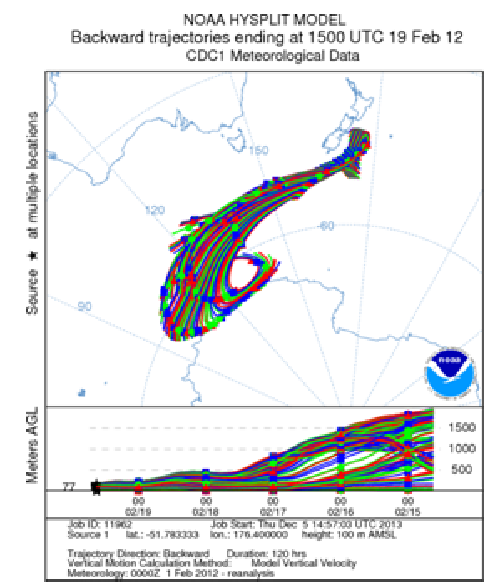

Figure S6G. 5-day back-trajectories of the seventh sample (from 18 to 19 February) collected during the oceanographic cruise on the R/V Italica during the austral summer Antarctica field 201112 on the Southern Ocean. The trajectories were computed for each 24-h sampling using a vertical velocity model at $100 \mathrm{~m}$ above sea level.

\section{References}

Barbaro, E., Zangrando, R., Moret, I., Barbante, C., Cescon, P., and Gambaro, A.: Free amino acids in atmospheric particulate matter of Venice, Italy, Atmospheric Environment, 45,

10.1016/j.atmosenv.2011.01.068, 2011.

Mandalakis, M., Apostolaki, M., and Stephanou, E. G.: Trace analysis of free and combined amino acids in atmospheric aerosols by gas chromatography-mass spectrometry, Journal of Chromatography A, 1217, 143150, 10.1016/j.chroma.2009.11.021, 2010.

Matsumoto, K., and Uematsu, M.: Free amino acids in marine aerosols over the western North Pacific Ocean, Atmospheric Environment, 39, 2163-2170, 10.1016/j.atmosenv.2004.12.022, 2005. 